\title{
Editorial:
}

\section{Some Critical Issues Related to Mental Health and Medication Use in the Elderly}

Two important current concerns in the health and well-being of older adults are raised by the research of Bourque et al. and reported in this issue of the Journal. The first is the relationship between physical health and depression, and the second is the use and misuse of medications among the elderly. The first issue has been addressed in studies referred to in the Bourque et al. paper that find a significant correlation between poor health and depression. However, there is also some evidence that individuals who suffer from depression show a great deal more illness over time (Wigdor \& Morris, 1977). This has important implications for aging since the research suggested that illnesses most frequently found in individuals with long histories of depressive episodes are those chronic conditions usually associated with aging, for example, cardiovascular disease, diabetes, and arthritis.

The second issue, that of medication use and misuse in the elderly raised in this paper is one that has been receiving increasing attention particularly from the health care sector. Because of the increasing number of older adults, and their increased likelihood of having one or more chronic medical conditions in later life the problem becomes critical.

The concern about how medication is prescribed and utilized in all age groups is based on the possibility of producing iatrogenic illness and dependencies. The elderly are particularly vulnerable for a variety of reasons. They are more likely to have chronic conditions which require medication over a long period of time, they frequently have more than one condition and tend to have more physician visits. Thus polypharmacy is frequently a fundamental problem. The elderly also metabolize and absorb medication differently and may have different attitudes and lifestyles. This situation can be complicated both by the prescribing behaviour of physicians, the compliance behaviour of elderly patients, the pattern of over-the-counter drug use and the kind of information available from physicians, pharmacists, and pharmaceutical companies. The type of disorders and the use of psychotherapeutic medication in individuals with varying states of health and the presence or absence of a mental disorder such as depression are other factors which can result in detrimental effects. This problem is highlighted in Bourque et al.'s paper. These investigators found that the prescription for psychotherapeutic medication was similar with or without the diagnosis of depression. Furthermore, they found that older individuals in poor health and those who are depressed use more medications which are not psychotherapeutic. Women were found in this study as in others to use more psychotherapeu- 
tic drugs than men.

The use of medication in the elderly is influenced not only by the multiple conditions of the elderly that need treatment but also by societal attitudes. These attitudes regarding the use of a "pill" or remedy for whatever ails one is shared by patients of all ages and by physicians. Older adults have been similarly socialized and because of their increased likelihood of developing physical conditions which may require some medication they become more susceptible to overuse or misuse. They are also confronted with many life transitions and social conditions which can cause stress, anxiety, fear and loneliness. Thus there is a higher probability that physicians will treat them for emotional distress with psychotherapeutic drugs rather than seek a cause and attempt to help with more effective coping strategies.

The overuse or misuse of medication is a health hazard for seniors as individuals but it is also a costly burden for the health care system. These are costs not only in terms of demands on benefit programs, where they exist, but also in the complications and ill effects of drugs themselves. When improperly used drugs may result in the need for care or hospitalization. Studies suggest that over 15 per cent of acute care hospitalizations of individuals over 65 are for iatrogenic illness mostly due to drug reactions.

The problems identified (Can. Coalition for Medication Use in the Elderly, 1991) as needing study and clarification are primarily how patients take drugs, how the drugs interact with one another, how eating habits and exercise levels affect drug absorption, and how the OTC drugs interact with prescribed drugs. In addition, the use of proper packaging, information and instructions as well as labelling have all been shown to influence proper medication usage. However, the prescribing behaviour of physicians bears investigation. Other research is required into the importance of the type of advertising of pharmaceutical manufacturers, the practice of pharmacists, and the attitudes and behaviour of patients.

The literature indicates that at least 40 per cent of adults over the age of 65 need some medication to carry on the activities of daily living and although Canadians over 65 make up 11 per cent of the population they consume 25 per cent of all drugs prescribed. Seventy per cent of this age group take at least one prescription drug and 13 per cent of males and 25 per cent of females take three or more. Bourque et al. found no significant difference between males and females in medication use except for the use of psychotherapeutic drugs. Sleeping sedation is also reported in the literature more frequently for females than males.

In terms of physicians' prescribing behaviour a number of factors must be considered. Knowledge of the effects of the medications being prescribed is often limited. Many physicians do not keep abreast of recent developments and are dependent on the sales representatives of the pharmaceutical companies. Furthermore, research in Canada on drugs and their effects are rarely carried out on the elderly nor on elderly women due to the difficulties in dealing with a multiplicity of variables and the vulnerability of the elderly. However, this leads to problems in terms of the determination of 
side effects and of appropriate dosages. Most of the drug research is originally carried out on relatively young males. Recently the United States has passed legislation requiring that drugs be tested on populations which will be major users. This will be useful in developing information for more appropriate use. Many physicians also are faced with busy schedules and may overlook asking patients about the various medications taken or the patients may not be willing to tell the doctor about medications possibly prescribed by other doctors. Over-the-counter drugs including vitamins, aspirins and laxatives may not be inquired for and/or may not be considered drugs or medication by the patient. Furthermore, physicians may not be very knowledgeable about the interaction of nutrition with prescribed medication or the impact of exercise on both nutrition and drugs. It has also been suggested that physicians feel under pressure from their patients to do something concrete and that the expectation of a prescription is so strong that a patient will visit another physician if one is not prescribed. There have been few studies of prescribing behaviour particularly with the elderly to substantiate this claim. In addition, time and/or lack of understanding of how to communicate information about the reason for medication and instructions for use often result in misunderstanding by the elderly patient and resulting misuse.

Patients, particularly elderly patients, have also been accused of expecting and wanting medication. They certainly may well expect that there is a "pill for every ill" since this is a societal pattern for all ages. Without clear instruction and explanation they may not understand why they have to take the drug as prescribed. Furthermore, without help in organizing medication, taking more than one drug a day and having to take them at different times can lead to confusion for patients. Some recent research by Denise Park (1991) indicated that some organizer containers are much more effective than others and some may even complicate the regime, such as those containers which dump a number of medications in a box for a day when they are to be taken at different times of the day. Labelling and clarity of instructions are other problems for the elderly patient. Again Park (1991) has found, at least for present-day elderly in the U.S., written verbal instructions rather than pictorial ones led to better compliance provided the print was large enough and the instructions clear. The literature on patient compliance is fairly extensive. However, one factor, the patient/physician relationship seems basic. The time needed to establish a relationship with elderly patients may be longer as there is a need to understand the history and lifestyle of the patient and to make sure instructions are understood. The remuneration system must take these factors into consideration. Changes may need to be instituted to allow physicians additional time with elderly patients and to compensate them appropriately for health promotion activities.

Research is necessary to understand better both the behaviour of patients and the behaviour of prescribing physicians. Public health nurses should be an integral link in this network, and clarification of their role is necessary. 
Furthermore, the responsibilities of pharmacists in information transfer and monitoring is critical. The pharmaceutical companies should be doing clinical trials on drugs using the populations for which they are primarily intended. There is also a need to develop more appropriate and sensitive advertising strategies. In general, creating awareness in the public of all ages and in all health care professionals of the problems related to medication use is a priority. A method for developing and prioritizing clear information must be developed. This is a very complex problem area and there is some danger in oversimplifying the underlying factors.

This editorial has emphasized the implications of the Bourque et al. paper in terms of misuse of medication among the elderly. However, it is important not to lose sight of the general health, including mental health, issues that this paper raises. The prevalence rate of depression among the elderly is not clear but depression and its often different manifestations in the elderly need further study. The interrelationship of health and well-being is a major concern. There is an increasing literature on this question but a great deal more work has to be done if the health care of the elderly is to be more effective. The goal should be better understanding so that illness is prevented and good health promoted. The mental health of the elderly has not been identified as a particular concern and mental health needs of the elderly have not been well met in the past. Both research and new care systems must be fostered to more adequately address this area. Underlying these problems is the health care delivery system. Social policy based on research into optimum health care delivery systems for an aging population will be needed if some of the above problems are to be tackled successfully.

\section{References}

Canadian Coalition on Medication Use of the Elderly. (1991). Proceedings of Research Conference in Medication Use. Ottawa.

Park, Denise C. (1991). Applied Cognitive Aging Research. In F.I.M. Craik \& T.A. Salthouse (Eds.), Handbook of Aging and Cognition. New York: Lawrence Erlbaum (in press).

Wigdor, Blossom T., \& Morris, Gerald (1977). A Comparison of Twenty-Year Medical Histories of Individuals with Depressive and Paranoid States. Journal of Gerontology, 32(2), 160-163.

Blossom T. Wigdor, University of Toronto 